\title{
Coordinate Finite Type Rotational Surfaces in Euclidean Spaces
}

\author{
B. K. Bayram, K. Arslan, N. Önen \& B. Bulca,
}

\begin{abstract}
Submanifolds of coordinate finite-type were introduced in 11. A submanifold of a Euclidean space is called a coordinate finite-type submanifold if its coordinate functions are eigenfunctions of $\Delta$. In the present study we consider coordinate finite-type surfaces in $\mathbb{E}^{4}$. We give necessary and sufficient conditions for generalized rotation surfaces in $\mathbb{E}^{4}$ to become coordinate finite-type. We also give some special examples.
\end{abstract}

\section{Introduction}

1 Let $\mathrm{M}$ be a connected $n$-dimensional submanifold of a Euclidean space $\mathbb{E}^{m}$ equipped with the induced metric. Denote $\Delta$ by the Laplacian of $M$ acting on smooth functions on $M$. This Laplacian can be extended in a natural way to $\mathbb{E}^{m}$ valued smooth functions on $M$. Whenever the position vector $x$ of $M$ in $\mathbb{E}^{m}$ can be decomposed as a finite sum of $\mathbb{E}^{m}$-valued non-constant functions of $\Delta$, one can say that $M$ is of finite type. More precisely the position vector $x$ of $M$ can be expresed in the form $x=x_{0}+\sum_{i=1}^{k} x_{i}$, where $x_{0}$ is a constant map $x_{1}, x_{2}, \ldots, x_{k}$ non-constant maps such that $\Delta x=\lambda_{i} x_{i}, \lambda_{i} \in \mathbb{R}$, $1 \leq i \leq k$. If $\lambda_{1}, \lambda_{2}, \ldots, \lambda_{k}$ are different, then $M$ is said to be of $k$-type. Similarly, a smooth map $\phi$ of an $n$-dimensional Riemannian manifold $M$ of $\mathbb{E}^{m}$ is said to be of finite type if $\phi$ is a finite sum of $\mathbb{E}^{m}$-valued eigenfunctions of $\Delta$ (2), 3]). For the position vector field $\vec{H}$ of $\mathrm{M}$ it is well known (see eg. 3]) that $\Delta x=-n \vec{H}$, which shows in particular that $M$ is a minimal submanifold in $\mathbb{E}^{m}$ if and only if its coordinate functions are harmonic. In 14 Takahasi proved that an n-dimensional submanifold of $\mathbb{E}^{m}$ is of 1-type (i.e., $\Delta x=\lambda x$ ) if and only if it is either a minimal submanifold of $\mathbb{E}^{m}$ or a minimal submanifold of some hypersphere of $\mathbb{E}^{m}$. As a generalization of T. Takahashi's condition, O. Garay considered in [9], submanifolds of Euclidean space whose position vector field $x$ satisfies the differential equation $\Delta x=A x$, for some $m \times m$ diagonal matrix $A$. Garay called such submanifolds coordinate finite type submanifolds. Actually coordinate finite type submanifolds are finite type submanifolds whose

\footnotetext{
${ }^{1} 2000$ AMS Mathematics Subject Classification. 53B25, 53C40, 53C42

Key words and phrases: Surfaces of restricted type, Rotational surface, Finite type surfaces
} 
type number $\mathrm{s}$ are at most $m$. Each coordinate function of a coordinate finite type submanifold $m$ is of 1-type, since it is an eigenfunction of the Laplacian [1].

In 8 by G. Ganchev and V. Milousheva considered the surface M generated by a $\mathrm{W}$-curve $\gamma$ in $\mathbb{E}^{4}$. They have shown that these generated surfaces are a special type of rotation surfaces which are introduced first by C. Moore in 1919 (see [13). Vranceanu surfaces in $\mathbb{E}^{4}$ are the special type of these surfaces [15].

This paper is organized as follows: Section 2 gives some basic concepts of the surfaces in $\mathbb{E}^{4}$. Section 3 tells about the generalized surfaces in $\mathbb{E}^{4}$. Further this section provides some basic properties of surfaces in $\mathbb{E}^{4}$ and the structure of their curvatures. In the final section we consider coordinate finite type surfaces in euclidean spaces. We give necessary and sufficient conditions for generalized rotation surfaces in $\mathbb{E}^{4}$ to become coordinate finite type.

\section{Basic Concepts}

Let $M$ be a smooth surface in $\mathbb{E}^{n}$ given with the patch $X(u, v):(u, v) \in D \subset \mathbb{E}^{2}$. The tangent space to $M$ at an arbitrary point $p=X(u, v)$ of $M$ span $\left\{X_{u}, X_{v}\right\}$. In the chart $(u, v)$ the coefficients of the first fundamental form of $M$ are given by

$$
\left.E=<X_{u}, X_{u}\right\rangle, F=\left\langle X_{u}, X_{v}\right\rangle, G=\left\langle X_{v}, X_{v}\right\rangle,
$$

where $\langle$,$\rangle is the Euclidean inner product. We assume that W^{2}=E G-F^{2} \neq$ 0 , i.e. the surface patch $X(u, v)$ is regular. For each $p \in M$, consider the decomposition $T_{p} \mathbb{E}^{n}=T_{p} M \oplus T_{p}^{\perp} M$ where $T_{p}^{\perp} M$ is the orthogonal component of $T_{p} M$ in $\mathbb{E}^{n}$. Let $\tilde{\nabla}$ be the Riemannian connection of $\mathbb{E}^{4}$. Given unit local vector fields $X_{1}, X_{2}$ tangent to $M$.

Let $\chi(M)$ and $\chi^{\perp}(M)$ be the space of the smooth vector fields tangent to $M$ and the space of the smooth vector fields normal to $M$, respectively. Consider the second fundamental map: $h: \chi(M) \times \chi(M) \rightarrow \chi^{\perp}(M)$;

$$
h\left(X_{i}, X_{j}\right)=\widetilde{\nabla}_{X_{i}} X_{j}-\nabla_{X_{i}} X_{j} \quad 1 \leq i, j \leq 2 .
$$

where $\widetilde{\nabla}$ is the induced. This map is well-defined, symmetric and bilinear.

For any arbitrary orthonormal normal frame field $\left\{N_{1}, N_{2}, \ldots, N_{n-2}\right\}$ of $M$, recall the shape operator $A: \chi^{\perp}(M) \times \chi(M) \rightarrow \chi(M)$;

$$
A_{N_{i}} X_{j}=-\left(\widetilde{\nabla}_{X_{j}} N_{k}\right)^{T}, \quad X_{j} \in \chi(M), 1 \leq k \leq n-2
$$

This operator is bilinear, self-adjoint and satisfies the following equation:

$$
\left\langle A_{N_{k}} X_{j}, X_{i}\right\rangle=\left\langle h\left(X_{i}, X_{j}\right), N_{k}\right\rangle=h_{i j}^{k}, 1 \leq i, j \leq 2 .
$$

The equation (21) is called Gaussian formula, and

$$
h\left(X_{i}, X_{j}\right)=\sum_{k=1}^{n-2} h_{i j}^{k} N_{k}, \quad 1 \leq i, j \leq 2
$$


where $c_{i j}^{k}$ are the coefficients of the second fundamental form.

Further, the Gaussian and mean curvature vector of a regular patch $X(u, v)$ are given by

$$
K=\sum_{k=1}^{n-2}\left(h_{11}^{k} h_{22}^{k}-\left(h_{12}^{k}\right)^{2}\right),
$$

and

$$
H=\frac{1}{2} \sum_{k=1}^{n-2}\left(h_{11}^{k}+h_{22}^{k}\right) N_{k},
$$

respectively, where $h$ is the second fundamental form of $M$. Recall that a surface $M$ is said to be minimal if its mean curvature vector vanishes identically [2]. For any real function $f$ on $M$ the Laplacian of $f$ is defined by

$$
\Delta f=-\sum_{i}\left(\widetilde{\nabla}_{e_{i}} \widetilde{\nabla}_{e_{i}} f-\widetilde{\nabla}_{\nabla_{e_{i}} e_{i}} f\right) .
$$

\section{Generalized Rotation Surfaces in $\mathbb{E}^{4}$}

Let $\gamma=\gamma(s): I \rightarrow \mathbb{E}^{4}$ be a W-curve in Euclidean 4-space $\mathbb{E}^{4}$ parametrized as follows:

$$
\gamma(v)=(a \cos c v, a \sin c v, b \cos d v, b \sin d v), 0 \leq v \leq 2 \pi,
$$

where $a, b, c, d$ are constants $(c>0, d>0)$. In [8] G. Ganchev and V. Milousheva considered the surface $M$ generated by the curve $\gamma$ with the following surface patch:

$$
X(u, v)=(f(u) \cos c v, f(u) \sin c v, g(u) \cos d v, g(u) \sin d v),
$$

where $u \in J, 0 \leq v \leq 2 \pi, f(u)$ and $g(u)$ are arbitrary smooth functions satisfying

$$
c^{2} f^{2}+d^{2} g^{2}>0 \text { and }\left(f^{\prime}\right)^{2}+\left(g^{\prime}\right)^{2}>0 .
$$

These surfaces are first introduced by C. Moore in [13, called general rotation surfaces.

We choose an orthonormal frame $\left\{e_{1}, e_{2}, e_{3}, e_{4}\right\}$ such that $e_{1}, e_{2}$ are tangent to $M$ and $e_{3}, e_{4}$ normal to $M$ in the following (see, [8]):

$$
\begin{aligned}
& e_{1}=\frac{X_{u}}{\left\|X_{u}\right\|}, e_{2}=\frac{X_{v}}{\left\|X_{u}\right\|} \\
& e_{3}=\frac{1}{\sqrt{\left(f^{\prime}\right)^{2}+\left(g^{\prime}\right)^{2}}}\left(g^{\prime} \cos c v, g^{\prime} \sin c v,-f^{\prime} \cos d v,-f^{\prime} \sin d v\right), \\
& e_{4}=\frac{1}{\sqrt{c^{2} f^{2}+d^{2} g^{2}}}(-d g \sin c v, d g \cos c v, c f \sin d v,-c f \cos d v) .
\end{aligned}
$$


Hence the coefficients of the first fundamental form of the surface are

$$
\begin{aligned}
E & =\left\langle X_{u}, X_{u}\right\rangle=\left(f^{\prime}\right)^{2}+\left(g^{\prime}\right)^{2} \\
F & =\left\langle X_{u}, X_{v}\right\rangle=0 \\
G & =\left\langle X_{v}, X_{v}\right\rangle=c^{2} f^{2}+d^{2} g^{2}
\end{aligned}
$$

where $\langle$,$\rangle is the standard scalar product in \mathbb{E}^{4}$. Since

$$
E G-F^{2}=\left(\left(f^{\prime}\right)^{2}+\left(g^{\prime}\right)^{2}\right)\left(c^{2} f^{2}+d^{2} g^{2}\right)
$$

does not vanish, the surface patch $X(u, v)$ is regular. Then with respect to the frame field $\left\{e_{1}, e_{2}, e_{3}, e_{4}\right\}$, the Gaussian and Weingarten formulas (2)-(3) of $M$ look like (see, [7]);

$$
\begin{aligned}
\tilde{\nabla}_{e_{1}} e_{1} & =-A(u) e_{2}+h_{11}^{1} e_{3}, \\
\tilde{\nabla}_{e_{1}} e_{2} & =A(u) e_{1}+h_{12}^{2} e_{4}, \\
\tilde{\nabla}_{e_{2}} e_{2} & =h_{22}^{1} e_{3}, \\
\tilde{\nabla}_{e_{2}} e_{1} & =h_{12}^{2} e_{4},
\end{aligned}
$$

and

$$
\begin{aligned}
\tilde{\nabla}_{e_{1}} e_{3} & =-h_{11}^{1} e_{1}+B(u) e_{4}, \\
\tilde{\nabla}_{e_{1}} e_{4} & =-h_{12}^{2} e_{2}-B(u) e_{3}, \\
\tilde{\nabla}_{e_{2}} e_{3} & =-h_{22}^{1} e_{2}, \\
\tilde{\nabla}_{e_{2}} e_{4} & =-h_{12}^{2} e_{1},
\end{aligned}
$$

where

$$
\begin{aligned}
A(u) & =\frac{c^{2} f f^{\prime}+d^{2} g g^{\prime}}{\sqrt{\left(f^{\prime}\right)^{2}+\left(g^{\prime}\right)^{2}}\left(c^{2} f^{2}+d^{2} g^{2}\right)}, \\
B(u) & =\frac{c d\left(f f^{\prime}+g g^{\prime}\right)}{\sqrt{\left(f^{\prime}\right)^{2}+\left(g^{\prime}\right)^{2}}\left(c^{2} f^{2}+d^{2} g^{2}\right)}, \\
h_{11}^{1} & =\frac{d^{2} f^{\prime} g-c^{2} f g^{\prime}}{\sqrt{\left(f^{\prime}\right)^{2}+\left(g^{\prime}\right)^{2}\left(c^{2} f^{2}+d^{2} g^{2}\right)}}, \\
h_{22}^{1} & =\frac{g^{\prime} f^{\prime \prime}-f^{\prime} g^{\prime \prime}}{\left(\left(f^{\prime}\right)^{2}+\left(g^{\prime}\right)^{2}\right)^{\frac{3}{2}}} \\
h_{12}^{2} & =\frac{c d\left(f^{\prime} g-f g^{\prime}\right)}{\sqrt{\left(f^{\prime}\right)^{2}+\left(g^{\prime}\right)^{2}\left(c^{2} f^{2}+d^{2} g^{2}\right)}} \\
h_{11}^{2} & =h_{22}^{2}=h_{12}^{1}=0 .
\end{aligned}
$$

are the differentiable functions. Using (6)-(7) with (14) one can get the following results; 
Proposition 1 [1] Let $M$ be a generalized rotation surface given by the parametrization (9), then the Gaussian curvature of $M$ is

$$
K=\frac{\left(c^{2} f^{2}+d^{2} g^{2}\right)\left(g^{\prime} f^{\prime \prime}-f^{\prime} g^{\prime \prime}\right)\left(d^{2} g f^{\prime}-c^{2} f g^{\prime}\right)-c^{2} d^{2}\left(g f^{\prime}-f g^{\prime}\right)^{2}\left(\left(f^{\prime}\right)^{2}+\left(g^{\prime}\right)^{2}\right)}{\left(\left(f^{\prime}\right)^{2}+\left(g^{\prime}\right)^{2}\right)^{2}\left(c^{2} f^{2}+d^{2} g^{2}\right)^{2}} .
$$

An easy consequence of Proposition 1 is the following.

Corollary 2 [1] The generalized rotation surface given by the parametrization (9) has vanishing Gaussian curvature if and only if the following equation

$\left(c^{2} f^{2}+d^{2} g^{2}\right)\left(g^{\prime} f^{\prime \prime}-f^{\prime} g^{\prime \prime}\right)\left(d^{2} g f^{\prime}-c^{2} f g^{\prime}\right)-c^{2} d^{2}\left(g f^{\prime}-f g^{\prime}\right)^{2}\left(\left(f^{\prime}\right)^{2}+\left(g^{\prime}\right)^{2}\right)=0$,

holds.

The following results are well-known;

Proposition 3 [1] Let $M$ be a generalized rotation surface given by the parametrization (9), then the mean curvature vector of $M$ is

$$
\begin{aligned}
\vec{H} & =\frac{1}{2}\left(h_{11}^{1}+h_{22}^{1}\right) e_{3} \\
& =\left(\frac{\left(c^{2} f^{2}+d^{2} g^{2}\right)\left(g^{\prime} f^{\prime \prime}-f^{\prime} g^{\prime \prime}\right)+\left(d^{2} g f^{\prime}-c^{2} f g^{\prime}\right)\left(\left(f^{\prime}\right)^{2}+\left(g^{\prime}\right)^{2}\right)}{2\left(\left(f^{\prime}\right)^{2}+\left(g^{\prime}\right)^{2}\right)^{3 / 2}\left(c^{2} f^{2}+d^{2} g^{2}\right)}\right) e_{3} .
\end{aligned}
$$

An easy consequence of Proposition 3 is the following.

Corollary 4 [1] The generalized rotation surface given by the parametrization (9) is minimal surface in $\mathbb{E}^{4}$ if and only if the equation

$$
\left(c^{2} f^{2}+d^{2} g^{2}\right)\left(g^{\prime} f^{\prime \prime}-f^{\prime} g^{\prime \prime}\right)+\left(d^{2} g f^{\prime}-c^{2} f g^{\prime}\right)\left(\left(f^{\prime}\right)^{2}+\left(g^{\prime}\right)^{2}\right)=0,
$$

holds.

Definition 5 The generalized rotation surface given by the parametrization

$$
f(u)=r(u) \cos u, g(u)=r(u) \sin u, c=1, d=1 .
$$

is called Vranceanu rotation surface in Euclidean 4-space $\mathbb{E}^{4}$ [15].

Remark 6 Substituting (15) into the equation given in Corollary 2 we obtain the condition for Vranceanu rotation surface which has vanishing Gaussian curvature;

$$
r(u) r^{\prime \prime}(u)-\left(r^{\prime}(u)\right)^{2}=0 .
$$

Further, and easy calculation shows that $r(u)=\lambda e^{\mu u},(\lambda, \mu \in R)$ is the solution is this second degree equation. So, we get the following result.

Corollary 7 [16] Let $M$ is a Vranceanu rotation surface in Euclidean 4-space. If $M$ has vanishing Gaussian curvature, then $r(u)=\lambda e^{\mu u}$, where $\lambda$ and $\mu$ are real constants. For the case, $\lambda=1, \mu=0, r(u)=1$, the surface $M$ is a Clifford torus, that is it is the product of two plane circles with same radius. 
Corollary 8 [1] Let $M$ is a Vranceanu rotation surface in Euclidean 4-space. If $M$ is minimal then

$$
r(u) r^{\prime \prime}(u)-3\left(r^{\prime}(u)\right)^{2}-2 r(u)^{2}=0 .
$$

holds.

Corollary 9 [1] Let $M$ is a Vranceanu rotation surface in Euclidean 4-space. If $M$ is minimal then

$$
r(u)=\frac{ \pm 1}{\sqrt{a \sin 2 u-b \cos 2 u}},
$$

where, $a$ and $b$ are real constants.

Definition 10 The surface patch $X(u, v)$ is called pseudo-umbilical if the shape operator with respect to $H$ is proportional to the identity (see, [2]). An equivalent condition is the following:

$$
<h\left(X_{i}, X_{j}\right), H>=\lambda^{2}<X_{i}, X_{j}>,
$$

where, $\lambda=\|H\|$. It is easy to see that each minimal surface is pseudo-umbilical.

The following results are well-known;

Theorem 11 [1] Let $M$ be a generalized rotation surface given by the parametrization (9) is pseudo-umbilical then

$$
\left(c^{2} f^{2}+d^{2} g^{2}\right)\left(g^{\prime} f^{\prime \prime}-f^{\prime} g^{\prime \prime}\right)-\left(d^{2} g f^{\prime}-c^{2} f g^{\prime}\right)\left(\left(f^{\prime}\right)^{2}+\left(g^{\prime}\right)^{2}\right)=0 .
$$

The converse statement of Theorem 11 is also valid.

Corollary 12 [1] Let $M$ be a Vranceanu rotation surface in Euclidean 4-space. If $M$ pseudo-umbilical then $r(u)=\lambda e^{\mu u}$, where $\lambda$ and $\mu$ are real constants.

\subsection{Coordinate Finite Type Surfaces in Euclidean Spaces}

In the present section we consider coordinate finite type surfaces in Euclidean spaces $\mathbb{E}^{n+2}$. A surface $M$ in Euclidean $m$-space is called coordinate finite type if the position vector field $X$ satisfies the differential equation

$$
\Delta X=A X,
$$

for some $m \times m$ diagonal matrix $A$. Using the Beltrami formula's $\Delta X=-2 \vec{H}$, with (7) one can get

$$
\Delta X=-\sum_{k=1}^{n}\left(h_{11}^{k}+h_{22}^{k}\right) N_{k}
$$

So, using (20) with (21) the coordinate finite type condition reduces to

$$
A X=-\sum_{k=1}^{n}\left(h_{11}^{k}+h_{22}^{k}\right) N_{k}
$$

For a non-compact surface in $\mathbb{E}^{4}$ O.J.Garay obtained the following: 
Theorem 13 [10] The only coordinate finite type surfaces in Euclidean 4-space $\mathbb{E}^{4}$ with constant mean curvature are the open parts of the following surfaces:

i) a minimal surface in $\mathbb{E}^{4}$,

ii) a minimal surface in some hypersphere $S^{3}(r)$,

iii) a helical cylinder,

$i v)$ a flat torus $S^{1}(a) \times S^{1}(b)$ in some hypersphere $S^{3}(r)$.

In 5] Chen-Dillen-Verstraelen-Vrancken proved the following theorem;

Theorem 14 [5] Assume $M$ is a surface in $\mathbb{E}^{4}$ that is immersed in $S^{3}(r)$ and has constant mean curvature. Then $M$ is of restricted type if and only if $M$ is one of the following:

i) an open part of a minimal surface of $S^{3}(r)$,

ii) an open part of $S^{2}\left(r^{\prime}\right)$ for $0<r^{\prime} \leq r$,

iii) an open part of the product of two circles $S^{1}(a) \times S^{1}(b)$, where $a, b>0$ and $a^{2}+b^{2}=r^{2}$.

\subsection{Surface of Revolution of Coordinate Finite Type}

A surface in $\mathbb{E}^{3}$ is called a surface of revolution if it is generated by a curve $C$ on a plane $\Pi$ when $\Pi$ is rotated around a straight line $L$ in $\Pi$. By choosing $\Pi$ to be the $x z$-plane and line $L$ to be the $x$ axis the surface of revolution can be parameterized by

$$
X(u, v)=(f(u), g(u) \cos v, g(u) \sin v),
$$

where $f(u)$ and $g(u)$ are arbitrary smooth functions. We choose an orthonormal frame $\left\{e_{1}, e_{2}, e_{3}\right\}$ such that $e_{1}, e_{2}$ are tangent to $M$ and $e_{3}$ normal to $M$ in the following:

$$
e_{1}=\frac{X_{u}}{\left\|X_{u}\right\|}, e_{2}=\frac{X_{v}}{\left\|X_{v}\right\|}, e_{3}=\frac{1}{\sqrt{\left(f^{\prime}\right)^{2}+\left(g^{\prime}\right)^{2}}}\left(g^{\prime},-f^{\prime} \cos v,-f^{\prime} \sin v\right),
$$

By covariant differentiation with respect to $e_{1}, e_{2}$ a straightforward calculation gives

$$
\begin{aligned}
\tilde{\nabla}_{e_{1}} e_{1} & =h_{11}^{1} e_{3}, \\
\tilde{\nabla}_{e_{2}} e_{2} & =-A(u) e_{1}+h_{22}^{2} e_{3}, \\
\tilde{\nabla}_{e_{2}} e_{1} & =A(u) e_{2}, \\
\tilde{\nabla}_{e_{1}} e_{2} & =0,
\end{aligned}
$$


where

$$
\begin{aligned}
A(u) & =\frac{g^{\prime}}{g \sqrt{\left(f^{\prime}\right)^{2}+\left(g^{\prime}\right)^{2}}} \\
h_{11}^{1} & =\frac{g^{\prime} f^{\prime \prime}-f^{\prime} g^{\prime \prime}}{\left(\left(f^{\prime}\right)^{2}+\left(g^{\prime}\right)^{2}\right)^{\frac{3}{2}}}, \\
h_{22}^{1} & =\frac{f^{\prime}}{g \sqrt{\left(f^{\prime}\right)^{2}+\left(g^{\prime}\right)^{2}}}, \\
h_{12}^{1} & =0 .
\end{aligned}
$$

are the differentiable functions. Using (6)-(17) with (26) one can get

$$
\vec{H}=\frac{1}{2}\left(h_{11}^{1}+h_{22}^{1}\right) e_{3}
$$

where $h_{11}^{1}$ and $h_{22}^{1}$ are the coefficients of the second fundamental form given in (26).

A surface of revolution defined by (23) is said to be of polynomial kind if $f(u)$ and $g(u)$ are polynomial functions in $u$ and it is said to be of rational kind if $f$ is a rational function in $g$, i.e., $f$ is the quotient of two polynomial functions in $g$ [4.

For finite type surfaces of revolution B.Y. Chen and S. Ishikawa obtained in [6] the following results;

Theorem 15 [6] Let $M$ be a surface of revolution of polynomial kind. Then $M$ is a surface of finite type if and only if either it is an open portion of a plane or it is an open portion of a circular cylinder.

Theorem 16 [6] Let $M$ be a surface of revolution of rational kind. Then $M$ is a surface of finite type if and only if $M$ is an open portion of a plane.

T. Hasanis and T. Vlachos proved the following.

Theorem 17 [11] Let $M$ be a surface of revolution. If $M$ has constant mean curvature and is of finite type then $M$ is an open portion of a plane, of a sphere or of a circular cylinder.

We proved the following result;

Lemma 18 Let $M$ be a surface of revolution given with the parametrization (23). Then $M$ is a surface of coordinate finite type if and only if diagonal matrix $A$ is of the form

$$
A=\left[\begin{array}{ccc}
a_{11} & 0 & 0 \\
0 & a_{22} & 0 \\
0 & 0 & a_{33}
\end{array}\right]
$$


where

$$
\begin{aligned}
& a_{11}=\frac{-g^{\prime}\left(g\left(g^{\prime} f^{\prime \prime}-f^{\prime} g^{\prime \prime}\right)+f^{\prime}\left(\left(f^{\prime}\right)^{2}+\left(g^{\prime}\right)^{2}\right)\right)}{f g\left(\left(f^{\prime}\right)^{2}+\left(g^{\prime}\right)^{2}\right)^{2}} \\
& a_{22}=a_{33}=\frac{f^{\prime}\left(g\left(g^{\prime} f^{\prime \prime}-f^{\prime} g^{\prime \prime}\right)+f^{\prime}\left(\left(f^{\prime}\right)^{2}+\left(g^{\prime}\right)^{2}\right)\right)}{g^{2}\left(\left(f^{\prime}\right)^{2}+\left(g^{\prime}\right)^{2}\right)^{2}}
\end{aligned}
$$

are differentiable functions.

Proof. Assume that the surface of revolution $M$ given with the parametrization (23) is of coordinate finite type. Then, from the equality (22)

$$
\Delta X=-\left(h_{11}^{1}+h_{22}^{1}\right) e_{3} .
$$

Further, substituting (26) into (30) and using (24) we get the result.

Remark 19 If the diagonal matrix $A$ is equivalent to a zero matrix then $M$ becomes minimal. So the surface of revolution $M$ is either an open portion of a plane or an open portion of a catenoid.

Minimal rotational surfaces are of coordinate finite type.

For the non-minimal case we obtain the following result;

Proposition 20 Let $M$ be a non-minimal surface of revolution given with the parametrization (23). If $M$ is coordinate finite type surface then

$$
f f^{\prime}+\lambda g g^{\prime}=0
$$

holds, where $\lambda$ is a nonzero constant.

Proof. Suppose that the entries of the diagonal matrix $A$ are real constants. Then using (29) one can get the following differential equations

$$
\begin{gathered}
\frac{-g^{\prime}\left(g\left(g^{\prime} f^{\prime \prime}-f^{\prime} g^{\prime \prime}\right)+f^{\prime}\left(\left(f^{\prime}\right)^{2}+\left(g^{\prime}\right)^{2}\right)\right)}{f g\left(\left(f^{\prime}\right)^{2}+\left(g^{\prime}\right)^{2}\right)^{2}}=c_{1} \\
\frac{f^{\prime}\left(g\left(g^{\prime} f^{\prime \prime}-f^{\prime} g^{\prime \prime}\right)+f^{\prime}\left(\left(f^{\prime}\right)^{2}+\left(g^{\prime}\right)^{2}\right)\right)}{g^{2}\left(\left(f^{\prime}\right)^{2}+\left(g^{\prime}\right)^{2}\right)^{2}}=c_{2} .
\end{gathered}
$$

where $c_{1}, c_{2}$ are nonzero real constants. Further, substituting one into another we obtain the result.

Example 21 The round sphere given with the parametrization $f(u)=r \cos u$, $g(u)=r \sin u$ satisfies the equality (31). So it is a coordinate finite type surface.

Example 22 The cone $f(u)=g(u)$ satisfies the equality (31). So it is a coordinate finite type surface. 


\subsection{Generalized Rotation Surfaces of Coordinate Finite Type}

In the present section we consider generalized rotation surfaces of coordinate finite type surfaces in Euclidean 4 -spaces $\mathbb{E}^{4}$.

We proved the following result;

Lemma 23 Let $M$ be a generalized rotation surface given with the parametrization (9). Then $M$ is a surface of coordinate finite type if and only if diagonal matrix $A$ is of the form

$$
A=\left[\begin{array}{cccc}
a_{11} & 0 & 0 & 0 \\
0 & a_{22} & 0 & 0 \\
0 & 0 & a_{33} & 0 \\
0 & 0 & 0 & a_{44}
\end{array}\right]
$$

where

$$
\begin{aligned}
& a_{11}=a_{22}=\frac{-g^{\prime}(u)\left(\left(d^{2} f^{\prime} g-c^{2} f g^{\prime}\right)\left(\left(f^{\prime}\right)^{2}+\left(g^{\prime}\right)^{2}\right)+\left(g^{\prime} f^{\prime \prime}-f^{\prime} g^{\prime \prime}\right)\left(c^{2} f^{2}+d^{2} g^{2}\right)\right)}{f(u)\left(\left(f^{\prime}\right)^{2}+\left(g^{\prime}\right)^{2}\right)^{2}\left(c^{2} f^{2}+d^{2} g^{2}\right)} \\
& a_{33}=a_{44}=\frac{f^{\prime}(u)\left(\left(d^{2} f^{\prime} g-c^{2} f g^{\prime}\right)\left(\left(f^{\prime}\right)^{2}+\left(g^{\prime}\right)^{2}\right)+\left(g^{\prime} f^{\prime \prime}-f^{\prime} g^{\prime \prime}\right)\left(c^{2} f^{2}+d^{2} g^{2}\right)\right)}{g(u)\left(\left(f^{\prime}\right)^{2}+\left(g^{\prime}\right)^{2}\right)^{2}\left(c^{2} f^{2}+d^{2} g^{2}\right)}
\end{aligned}
$$

are differentiable functions and $h_{11}^{1}, h_{22}^{1}$ the coefficients of the second fundamental form given in (14).

If the matrix $A$ is a zero matrix then $M$ becomes minimal. So minimal rotational surfaces are of coordinate finite type.

We prove the following result.

Proposition 24 Let $M$ be a generalized rotation surface given by the parametrization (9). If $M$ is a coordinate finite type then

$$
f f^{\prime}=c g g \prime
$$

holds, where, $c$ is a real constant.

Proof. Suppose that the entries of the diagonal matrix $A$ are real constants. Then, substituting the first equation in (33) into second one we get the result.

An easy consequence of Proposition 24 is the following.

Corollary 25 Let $M$ be a Vranceanu rotation surface in Euclidean 4-space. If $M$ is a coordinate finite type, then

$$
r r^{\prime}\left(\cos ^{2} u-c \sin ^{2} u\right)=r^{2} \cos u \sin u(1+c)
$$

holds, where, $c$ is a real constant. 
We obtain the following result;

Theorem 26 Let $M$ be a Vranceanu rotation surface in Euclidean 4-space. Then $M$ is of restricted type if and only if $M$ is one of the following:

i) an open part of a Clifford torus,

ii) a minimal surface given with the parametrization (17).

iii) a surface given with the parametrization

$$
r(u)=\frac{ \pm \lambda}{\sqrt{(1+c) \cos 2 u+(1-c)}}, c \neq 1
$$

where, $\lambda$ and $c$ are real constants.

In 12 C. S. Houh investigated Vranceanu rotation surfaces of finite type and proved the following

Theorem 27 [12] A flat Vranceanu rotation surface in $\mathbb{E}^{4}$ is of finite type if and only if it is the product of two circles with the same radius, i.e. it is a Clifford torus.

\section{References}

[1] K. Arslan, B. Bayram, B. Bulca and G. Öztürk, Generalized Rotation Surfaces in $\mathbb{E}^{4}$, Results. Math. 61 (2012), 315-327

[2] B.Y. Chen, Geometry of Submanifolds and Its Applications, Science University of Tokyo, Tokyo, (1981).

[3] B.Y. Chen, Total Mean Curvature and Submanifolds of Finite Type, World Scientific, Singapur, (1984).

[4] B.Y. Chen, A Report on Submanifolds of Finite type, Soochow J. Math. 22(1996), 117-337.

[5] B.Y. Chen, F. Dillen, L. Verstraelen and L. Vrancken, Submanifolds of Restricted Type. Journal of Geometry, 46 (1993), 20-32.

[6] B. Y. Chen and S. Ishikawa, On Classification of Some Surfaces of Revolution of Finite Type, Tsukuba J. Math. 17 (1993), 287-298.

[7] U. Dursun and N.C. Turgay, General Rotation Surfaces in Euclidean Space $\mathbb{E}^{4}$ with Pointwise 1-type Gauss Map, Math. Commun. 17(2012), 71-81.

[8] G. Ganchev and V. Milousheva, On the Theory of Surfaces in the Fourdimensional Euclidean Space, Kodai Math. J., 31 (2008), 183-198.

[9] O.J. Garay, An Extension of Takahashi's Theorem, Geom. Dedicate 34 (1990), 105-112. 
[10] O.J. Garay, Orthogonal Surfaces with Constant Mean Curvature in the Euclidean Space, Ann. Global Anal. Geom.12(1994), 79-86.

[11] T. Hasanis and T Vlachos, Coordinate finite-type Submanifolds, Geom. Dedicata, 37(1991),155-165.

[12] C. S. Houh, Rotation Surfaces of Finite Type, Algebras Groups and Geometries, 7(1990), 199-209.

[13] C. Moore, Surfaces of Rotations in a Space of Four Dimensions. Ann. Math., 21(2) (1919) 81-93.

[14] Takahashi, T., Minimal Immersions of Riemannian Manifolds, J. Math. Soc. Japan18 (1966), 380-385.

[15] G. Vranceanu, Surfaces de Rotation dans $E^{4}$, Rev. Roum. Math. Pure Appl. XXII(6), 857-862 (1977).

[16] D. W. Yoon, Some Properties of the Clifford Torus as Rotation Surfaces. Indian J. Pure Appl. Math., 34(2003), 857-862.

Bengü (Kılıç) Bayram

Department of Mathematics

Balıkesir University

Balıkesir, Turkey

e-mail: benguk@balikesir.edu.tr

Kadri Arslan \& Betül Bulca

Department of Mathematics

Uludağ University

16059, Bursa, Turkey

e-mail: arslan@uludag.edu.tr; bbulca@uludag.edu.tr

Nergiz Önen

Department of Mathematics

Çukurova University

Adana, Turkey

e-mail: nonen@cu.edu.tr 